\title{
Atributos físicos de dois Latossolos afetados pela compactação do solo
}

\author{
Sérgio R. Silva ${ }^{1}$, Nairam F. Barros ${ }^{2}$ \& Liovando M. Costa ${ }^{2}$
}

\begin{abstract}
RESUMO
Um experimento foi realizado para avaliar os efeitos da compactação sobre os atributos físicos de dois solos: um Latossolo Vermelho-Amarelo (LVA) oxídico-gibbsítico, e um Latossolo Amarelo (LA) caulinítico. O ensaio consistiu de oito pressões de compactação (0, 60, 120, 240, 360, 540, 720 e 900 kPa). Cada unidade experimental se compunha de anel de PVC com $6 \mathrm{~cm}$ de altura e 4,55 cm de diâmetro interno, na qual foram avaliadas: condutividade hidráulica, microporosidade, macroporosidade, porosidade total, resistência do solo à penetração e agregados estáveis em água. A retenção de água a 0,01 e 1,5 MPa, foi avaliada em amostras de solos compactados em anéis de PVC com 1,9 cm de altura e 4,55 cm de diâmetro interno. $\mathrm{O}$ solo caulinítico foi mais sensível à compactação que o solo oxídico-gibbsítico. O incremento da microporosidade com a compactação aumentou a retenção de água no solo, nas tensões de 0,01 e 1,5 MPa; no entanto, este aumento não se expressou em termos de conteúdo de água disponível no solo. A condutividade hidráulica e a resistência à penetração foram as propriedades do solo mais sensíveis aos efeitos da compactação.
\end{abstract}

Palavras-chave: condutividade hidráulica, porosidade do solo, resistência à penetração, retenção de água no solo

\section{Physical attributes of two Oxisols affected by soil compaction}

\begin{abstract}
An experiment was carried out in order to evaluate the effects of soil compaction on the physical characteristics of two soils: a oxidic-gibbsitic Red Yellow Oxisol (LVA) and a caulinitic Yellow Oxisol (LA). The experiment consisted of eight levels of pressure applied to compact the soil $(0,60,120,240,360,540,720$ e $900 \mathrm{kPa})$. The experimental unit was composed of a PVC pipe with $6 \mathrm{~cm}$ in height and $4.55 \mathrm{~cm}$ of internal diameter to evaluate: hydraulic conductivity, microporosity, macroporosity, total porosity, soil penetration resistence and water-stable aggregates. Soil water retention at 0.01 and $1.5 \mathrm{MPa}$ was evaluated using the samples compacted in the PVC pipe with $1.9 \mathrm{~cm}$ in height and $4.55 \mathrm{~cm}$ of internal diameter. The caulinitic soil was more sensitive to soil compaction than the to oxidic-gibbsitic soil. The increase of microporosity with soil compaction increased the soil water retention at 0.01 and $1.5 \mathrm{MPa}$, however it did not affect available water holding capacity. The properties of soil which have higher sensibility to effects of soil compaction were hydraulic conductivity and penetration resistence.
\end{abstract}

Key words: hydraulic conductivity, soil porosity, penetration resistence, soil water retention 


\section{INTRODUÇÃO}

Compactação do solo refere-se à compressão do solo não saturado durante a qual existe um aumento de sua densidade em conseqüência da redução do seu volume. Esta operação resulta na expulsão de ar dos poros, causando um rearranjamento das partículas, tornando o solo mais denso e conseqüente redução da porosidade (Curi et al., 1993; Dias Júnior \& Pierce, 1996). A elevação da densidade do solo prejudica o desenvolvimento das plantas, ocasiona aumento da resistência mecânica à penetração de raízes, altera a movimentação de água e nutrientes e a difusão de oxigênio e outros gases, levando ao acúmulo de $\mathrm{CO}_{2}$ e de fitotoxinas (Marschner, 1995; Nadian et al., 1996; Borges et al., 1997; Mapfumo et al., 1998; Ishaq et al., 2001).

As propriedades físicas do solo, que passam por inúmeras mudanças provocadas pela compactação, indicam a sensibilidade do solo aos impactos de técnicas de manejo e fornecem informação sobre a sua capacidade em manter determinada função, como reter e disponibilizar água e nutrientes às plantas (McQueen \& Shepherd, 2002); assim, avaliações como: agregados estáveis em água (Silva et al., 2000; Beutler et al., 2001), densidade, porosidade e condutividade hidráulica do solo (Marsili et al., 1998; Ishaq et al., 2001; McQueen \& Shepherd, 2002), têm sido utilizadas como indicadores físicos da qualidade do solo, viabilizando estratégias para avaliar alterações de suas propriedades.

A redução da infiltração de água no solo, decorrente da compactação da camada superficial, pode resultar em aumento do escoamento superficial e erosão (Kayombo \& Lal, 1994). $\mathrm{O}$ aumento da resistência do solo à penetração pode ser restritivo ao crescimento radicular, quando acima de valores, que variam de 1,5 a 4,0 MPa (Oussible et al., 1992; Grant \& Lafond, 1993; Arshad et al., 1996).

Os objetivos do trabalho foram avaliar os efeitos da compactação sobre propriedades físicas de dois Latossolos com mineralogias distintas e definir as características físicas do solo, que apresentam maior sensibilidade para avaliação desses efeitos.

\section{MATERIAL E MÉTODOS}

Foram utilizados dois solos com mineralogia distinta, um Latossolo Vermelho-Amarelo (LVA) oxídico-gibbsítico do município de Santa Maria de Itabira, MG (19² 23' 58" S e 42 54' 12" W) e um Latossolo Amarelo (LA) caulinítico de San-

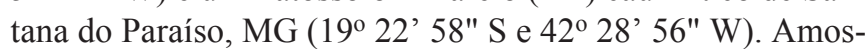
tras dos solos foram coletadas em fevereiro de 2003 em povoamentos de eucalipto, na profundidade de $0-15 \mathrm{~cm}$, postas para secar ao ar, passadas em peneira de $4 \mathrm{~mm}$ de malha e homogeneizadas, para posterior análises química e física (Tabela 1).

Dois ensaios foram desenvolvidos, um para cada solo, cada qual consistindo de oito tratamentos, correspondendo a oito pressões de compactação: 0, 60, 120, 240, 360, 540, 720 e 900 $\mathrm{kPa}$. Os tratamentos, com cinco repetições, totalizando 40 unidades experimentais para cada solo, foram dispostos em
Tabela 1. Caracterização do solo Latossolo Vermelho Amarelo (LVA) e Latossolo Amarelo (LA) utilizados no

\begin{tabular}{lcc}
\hline Característica & LVA & LA \\
\hline $\left.\mathrm{COT}(\mathrm{dag} \mathrm{kg})^{-1}\right)^{(1)}$ & 2,76 & 2,20 \\
$\mathrm{~N}$-total $\left(\mathrm{dag} \mathrm{kg}^{-1}\right)^{(2)}$ & 0,15 & 0,13 \\
\hline Equivalente de umidade $\left(\mathrm{kg} \mathrm{kg}^{-1}\right)$ & 0,2 & 0,2 \\
\hline Densidade do solo $\left(\mathrm{kg} \mathrm{dm}^{-3}\right)$ & 1,05 & 1,10 \\
\hline Argila $\left(\mathrm{g} \mathrm{kg}^{-1}\right)$ & 470 & 510 \\
\hline Silte $\left(\mathrm{g} \mathrm{kg}^{-1}\right)$ & 90 & 40 \\
\hline Areia $\left(\mathrm{g} \mathrm{kg}^{-1}\right)$ & 440 & 450 \\
\hline $\mathrm{Classe} \mathrm{textural}$ & Argila & Argila \\
\hline $\mathrm{SiO}_{2}\left(\mathrm{~g} \mathrm{~kg}^{-1}\right)^{(3)}$ & 71,29 & 135,19 \\
$\mathrm{Al}_{2} \mathrm{O}_{3}\left(\mathrm{~g} \mathrm{~kg}^{-1}\right)^{(3)}$ & 172,93 & 139,99 \\
$\mathrm{Fe}_{2} \mathrm{O}_{3}\left(\mathrm{~g} \mathrm{~kg}^{-1}\right)^{(3)}$ & 60,64 & 44,64 \\
\hline
\end{tabular}

1 Carbono orgânico total (Yeomans \& Bremner, 1988)

2 Nitrogênio total (Bremner \& Mulvaney, 1982)

${ }^{3}$ Elementos no extrato do ataque sulfúrico (EMBRAPA, 1997)

delineamento de blocos casualizados. Cada unidade experimental se compunha de anel de PVC com $6 \mathrm{~cm}$ de altura e $4,55 \mathrm{~cm}$ de diâmetro interno, com volume de $97,6 \mathrm{~cm}^{3}$.

Calculou-se a massa de cada solo para ocupar o volume de $97,6 \mathrm{~cm}^{3}$ e atingir as densidades de 1,05 e $1,10 \mathrm{~kg} \mathrm{dm}^{-3}$, respectivamente, nos LVA e LA, a seguir, as amostras de solo foram umedecidas $\left(0,20 \mathrm{~kg} \mathrm{~kg}^{-1}=100 \%\right.$ do equivalente de umidade), acondicionadas nos vasos e compactadas utilizando-se uma prensa CBR equipada com anel dinamométrico; depois foi calculada a densidade do solo resultante da compactação, por meio do novo volume ocupado.

Para a avaliação da condutividade hidráulica, os tubos de PVC foram adaptados como permeâmetros de carga constante, sendo a condutividade hidráulica obtida pela aplicação da equação de Darcy, após os valores de fluxo atingirem a estabilidade (EMBRAPA, 1997). Nessas amostras foi mensurada a microporosidade pelo método da mesa de tensão e calculadas a porosidade total e a macroporosidade (EMBRAPA, 1997). Após equilíbrio da umidade do solo a 0,006 MPa na mesa de tensão, mediu-se, a resistência do solo à penetração utilizando-se um penetrômetro eletrônico com velocidade constante de penetração de $2 \mathrm{~cm} \mathrm{~min}^{-1}$ e um cone com diâmetro de base de $4 \mathrm{~mm}$ e semi-ângulo de $30^{\circ}$. Este penetrômetro estava equipado com um atuador linear e célula de carga de $20 \mathrm{~kg}$ acoplado a um microcomputador para aquisição dos dados. No centro geométrico de cada amostra, na profundidade de 5 a $55 \mathrm{~mm}$, uma leitura de resistência foi obtida a cada $0,31 \mathrm{~mm}$, perfazendo o total de 160 leituras, as quais foram utilizadas para calcular a média da resistência do solo à penetração de cada amostra.

O procedimento de compactação do solo, já descrito, foi realizado novamente para posterior análise de agregados estáveis em água (EMBRAPA, 1997) e obtenção do diâmetro médio geométrico, segundo Kemper \& Rosenau (1986).

$A$ retenção de água no solo às tensões de 0,01 e 1,5 $\mathrm{MPa}$ de tensão, foi avaliada utilizando-se anel de PVC com 1,9 cm de altura, 4,55 cm de diâmetro interno e volume útil de 30,9 $\mathrm{cm}^{3}$. Realizou-se a compactação do solo de modo idêntico ao 
descrito antes; no entanto, com 6 blocos (repetições). A seguir, as amostras de solo (individualmente por bloco com todos os tratamentos) foram saturadas e transferidas para o aparelho extrator de Richards, onde foram submetidas sucessivamente às tensões de 0,01 e 1,5 $\mathrm{MPa}$ até atingirem a drenagem máxima (EMBRAPA, 1997). A água disponível no solo (ADS) foi obtida pela diferença entre a água retida a 0,01 e 1,5 $\mathrm{MPa}$.

Os resultados foram submetidos, individualmente para cada solo, a análise de variância, de correlação e de regressão. Para a escolha dos modelos de regressão, os coeficientes foram testados pelo teste $\mathrm{F}$, com base no quadrado médio do resíduo da ANOVA do experimento. Finalmente, para dois ou mais modelos com coeficientes significativos, a seleção foi feita com base no maior coeficiente de determinação $\left(\mathrm{R}^{2}\right)$.

\section{RESULTADOS E DISCUSSÃO}

Ocorreu aumento da densidade do solo $\left(D_{S}\right)$ em resposta às pressões de compactação (Figura 1). $\mathrm{O}$ aumento foi de 27 e $37 \%$ com maior pressão $(900 \mathrm{kPa})$, respectivamente, no LVA e LA. O menor aumento $\mathrm{D}_{\mathrm{S}}$ no LVA pode ser devido à sua mineralogia, pois os óxidos de Fe e Al, predominantes em sua composição, se associam às argilas silicatadas, formando agregados pequenos e extremamente estáveis com maior resistência à deformação, quando sujeitos a pressão. Por sua vez, no LA a caulinita se modela e/ou se deforma mais facilmente que os óxidos, facilitando o adensamento do solo.
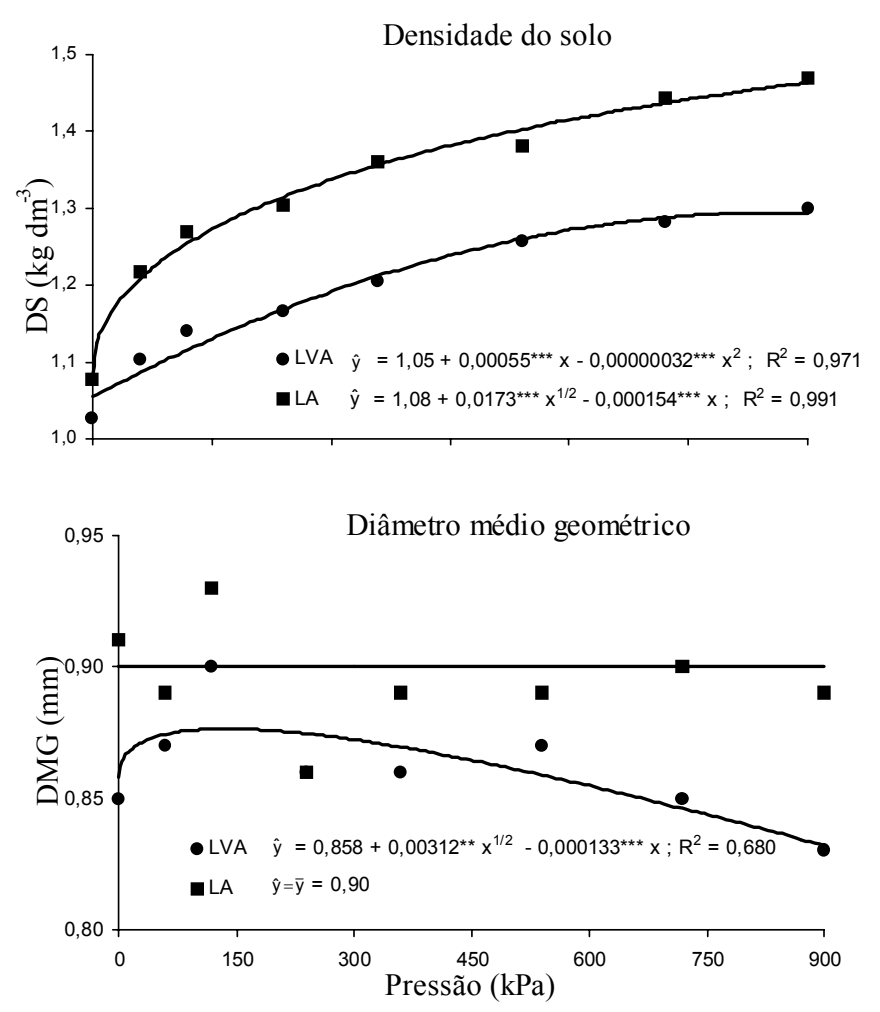

Figura 1. Densidade e diâmetro médio geométrico dos agregados de dois Latossolos compactados com diferentes pressões
O diâmetro médio geométrico (DMG) dos agregados no LVA aumentou com a compactação do solo em níveis intermediários de pressão, decrescendo a seguir para maiores pressões (Figura 1). O aumento atingiu 6\% no LVA (pressão = $120 \mathrm{kPa}$ ). Pode-se atribuir este resultado à provável agregação mecânica do LVA por efeito da pressão exercida pela compactação, levando à junção de partículas sob alta umidade no solo. $\mathrm{O}$ DMG não revelou ser bom indicador dos efeitos da compactação para ambos os solos, principalmente para o LA.

A compactação acarretou decréscimo da porosidade total de 17 e $23 \%$, refletindo em redução da macroposidade de 53 e $67 \%$, e aumento da microposidade de 35 e $32 \%$, respectivamente, nos LVA e LA, na pressão de $900 \mathrm{kPa}$ (Figura 2). Constatou-se que a macroporosidade apresentou correlação significativa $(\mathrm{p}<0,001)$ e negativa com a densidade e a microporosidade ( $r=-0,96$ no LVA e $r=-0,99$ no LA), ou seja, a compactação proporcionou modificação do sistema poroso, transformando ambientes de macroporos em microporos.

A tendência dos dados foi semelhante à observada por Marsili et al. (1998) que verificaram decréscimo da macroporosidade na camada superficial $(0-10 \mathrm{~cm})$ depois de uma passada de dois tratores, cujo efeito foi intensificado por 4 passadas das máquinas. Wagger \& Denton (1989) observaram que a porosidade total na área de solo trafegado foi $21 \%$ menor que no local não trafegado.

A compactação proporcionou aumento da retenção de água pelo solo a 0,01 e 1,5 MPa de tensão, tanto em base volumétrica $\left(\theta_{\mathrm{vol}}\right)$ como gravimétrica $\left(\theta_{\text {grav }}\right)$ (exceção para $0,01 \mathrm{MPa}$ em base gravimétrica no LA) (Figura 2). O aumento da $\theta_{\mathrm{vol}}$ atingiu 38 e $43 \%$ a $0,01 \mathrm{MPa}$, e 51 e $65 \%$ a $1,5 \mathrm{MPa}$, respectivamente, nos LVA e LA (pressão $=900 \mathrm{kPa}$ ). A $\theta_{\text {grav }}$ a 0,01 MPa aumentou 7\% no LVA (pressão $=540 \mathrm{kPa}$ ) e decresceu $8 \%$ no LA (pressão $=720 \mathrm{kPa}$ ), sendo que a 1,5 MPa ocorreu aumento de $11 \%$ da $\theta_{\text {grav }}$ nos dois solos, por efeito da maior compactação $(900 \mathrm{kPa})$. O aumento da retenção de água na tensão de 1,5 MPa foi maior, indicando que a modificação do sistema poroso pela compactação beneficiou especialmente a formação de poros capilares menores, onde a água é retida com mais energia.

A microporosidade apresentou correlação significativa $(\mathrm{p}$ $<0,001)$ e positiva com a $\theta_{\mathrm{vol}}$ a $0,01 \mathrm{MPa}(\mathrm{r}=0,75$ no LVA; e $\mathrm{r}=0,87$ no LA) e $1,5 \mathrm{MPa}(\mathrm{r}=0,76$ no LVA; e $\mathrm{r}=0,92$ no LA), e também com a $\theta_{\text {grav }}(p<0,005)$ a $1,5 \mathrm{MPa}(r=0,41$ no LVA; $\mathrm{r}=0,40$ no LA). O aumento da microporosidade com a compactação (Figura 2) promoveu incremento da retenção de água no solo nas tensões de 0,01 e 1,5 MPa, tanto em base volumétrica como em base gravimétrica, ou seja, foi notório o aumento do volume de microporos (mais água armazenada por unidade de volume de solo) e da força de retenção (mais água armazenada por unidade de massa de solo).

O aumento da retenção de água a 0,01 e 1,5 MPa não resultou em maior disponibilidade de água às plantas, pois não houve diferença no conteúdo de água disponível no solo (ADS) por efeito da compactação, exceção para $\theta_{\text {grav }}$ no LA, cuja redução da ADS foi de 48\%, após uma compactação de $900 \mathrm{kPa}$ (Figura 2). Esses resultados não corroboram com a afirmação de Greacen \& Sands (1980), de que o maior conteú- 

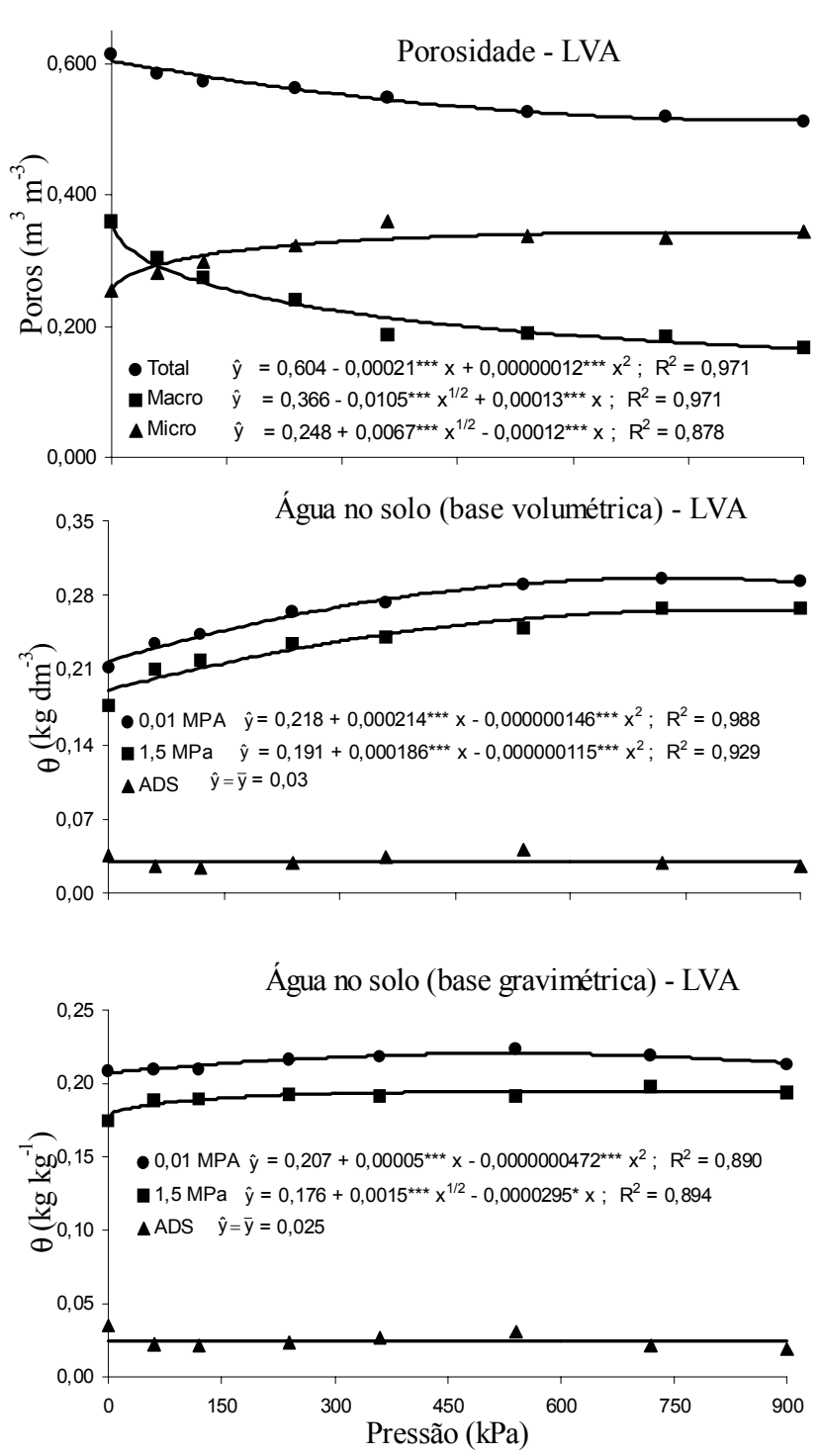
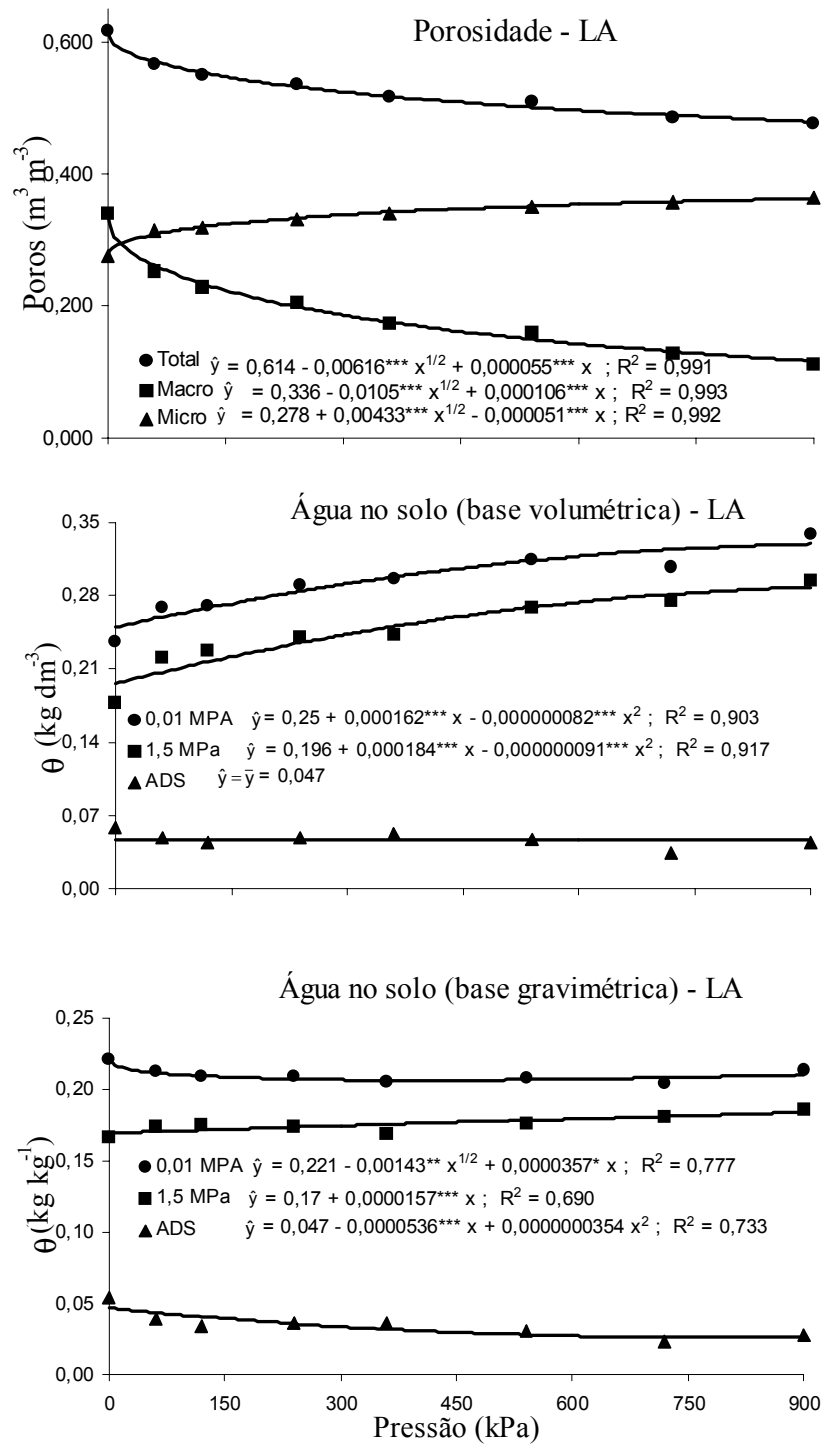

Figura 2. Porosidade, retenção de água a 0,01 e 1,5 MPa e água disponível no solo (ADS) de dois Latossolos compactados com diferentes pressões
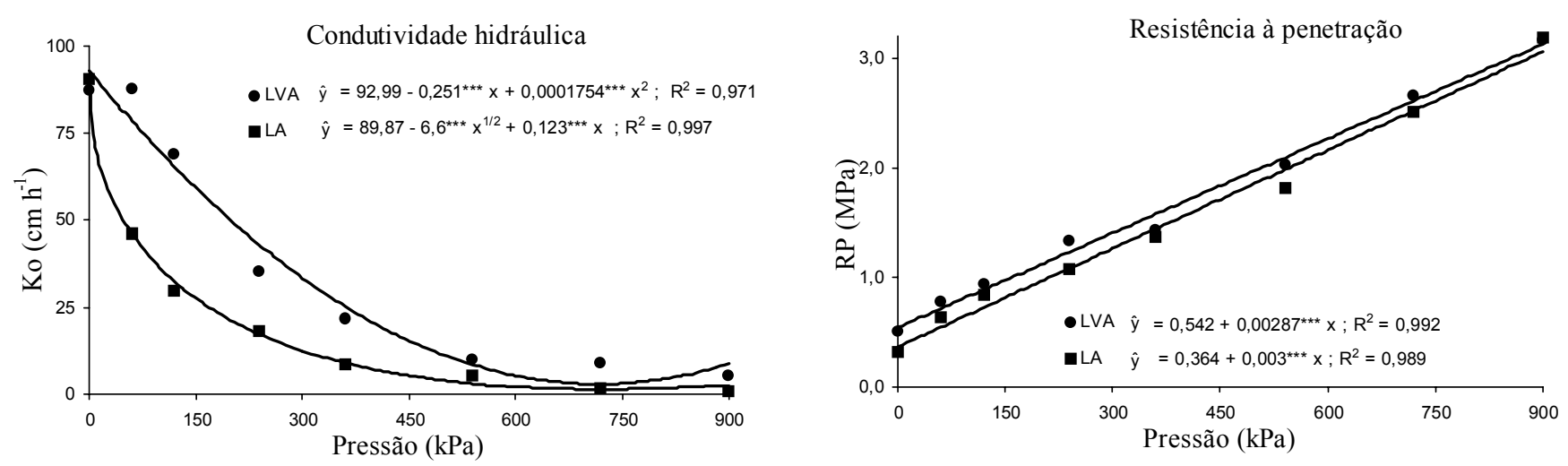

Figura 3. Condutividade hidráulica e resistência à penetração de dois Latossolos compactados com diferentes pressões

do volumétrico de água na capacidade de campo (0,01 MPa), em solo compactado, aumenta o volume de água por unidade de volume de solo que é disponível para as raízes das plantas.

A condutividade hidráulica $\left(\mathrm{K}_{0}\right)$ decresceu em resposta à compactação do solo (Figura 3 ). A redução da $\mathrm{K}_{0}$, em rela- ção à testemunha não compactada, atingiu 94 e 99\%, respectivamente, nos LVA e LA, enquanto a $\mathrm{K}_{0}$ apresentou correlação significativa $(p<0,001)$ e negativa com a densidade $(\mathrm{r}=-0,80$ no LVA; e $\mathrm{r}=-0,86$ no LA) e com a resistência à penetração ( $r=-0,74$ no LVA; e $r=-0,69$ no LA) e positiva 
com a macroporosidade ( $r=0,81$ no LVA; e $r=0,87$ no LA).

Nas pressões mais baixas (60 a $360 \mathrm{kPa}$ ) a $\mathrm{K}_{0}$ decresceu mais no LA, o que pode ser atribuído à mineralogia deste solo, pois a caulinita se modela e/ou deforma mais facilmente que os óxidos, quando sujeita a pressão, facilitando a formação de selamento do solo e conseqüente redução da $\mathrm{K}_{0}$. Por sua vez, o LVA possui predomínio de óxidos de Fe e de Al, apresentando agregados pequenos e extremamente estáveis devido à associação desses óxidos com argilas silicatadas; isto confere, ao LVA, maior resistência à deformação, quando sujeito a pressão, o que explica a menor redução da $\mathrm{K}_{0}$ em relação ao LA quando submetido a pressões de 60 a $360 \mathrm{kPa}$; mas pressões superiores a $540 \mathrm{kPa}$ foram altas o suficiente para igualar o grau de deformação desses solos e também a redução da $\mathrm{K}_{0}$.

A redução da condutividade hidráulica deve estar relacionada à reorganização do sistema poroso, ou seja, da distribuição do tamanho, elongação e continuidade vertical dos poros, ocasionada pela compactação do solo, como observado por Marsili et al. (1998). Segundo Silva et al. (1986) a redução da $\mathrm{K}_{0}$ é atribuída ao decréscimo da macroporosidade com o aumento da densidade, uma vez que o movimento de água no solo está diretamente relacionado com a porosidade de drenagem. Dependendo do tipo de solo, um pequeno aumento da densidade, causado pela compactação, pode diminuir a taxa de infiltração em até 80\% (Oliveira et al., 1998).

A condutividade hidráulica de um solo é um atributo de expressiva importância à dinâmica e ao armazenamento de água no solo. Solos com baixa $\mathrm{K}_{0}$ são mais vulneráveis à erosão, devido à maior quantidade de água que permanece na superfície do solo e, conseqüentemente, maior escoamento superficial (Cerdà, 1996).

A resistência do solo a penetração (RP) aumentou linearmente com a compactação (Figura 3). O aumento da RP foi de 6,3 e 10,3 vezes, respectivamente, nos LVA e LA, nos solos compactados com maior pressão $(900 \mathrm{kPa})$. Os valores máximos de resistência a penetração foram de 3,16 e 3,19 MPa, respectivamente, nos LVA e LA. A compactação, como evidenciada pelo aumento da resistência a penetração, reduz a penetrabilidade de raízes no solo (Unger \& Kaspar, 1994). É oportuno destacar que qualquer resistência a penetração superior a $2 \mathrm{MPa}$ pode reduzir o crescimento e o desenvolvimento radicular (Oussible et al., 1992; Martino \& Shaykewich, 1994; Ishaq et al., 2001). Desta forma estima-se, no presente estudo, que a pressão de compactação limite (para não prejudicar o crescimento de raízes) é $540 \mathrm{kPa}$, na qual a RP foi 2,02 e 1,81 MPa, respectivamente, nos LVA e LA.

Constatou-se que a resistência a penetração apresentou correlação significativa $(\mathrm{p}<0,001)$ e positiva com a densidade $(r=0,91$ e 0,90$)$ e com a microporosidade $(r=0,68$ e 0,87$)$; e negativa com a porosidade total $(\mathrm{r}=-0,91$ e $-0,90)$, com a macroporosidade $(r=-0,83$ e $-0,89)$ e com a $\mathrm{K}_{0}(\mathrm{r}=-0,74$ e 0,69 , respectivamente, nos LVA e LA). Esses resultados estão de acordo com os dados encontrados por Pagliai et al. (1992) e Marsili et al. (1998) que, em experimentos de compactação do solo pelo trânsito de máquinas, também obtiveram boa correlação negativa entre resistência a penetração e macroporosidade do solo. Estão coerentes também com Beu- tler et al. (2001) que encontraram correlação significativa ( $\mathrm{p}<$ $0,05)$ e negativa entre resistência a penetração e $K_{0}(r=-0,98)$.

\section{CONCLUSÕES}

1. Solos cauliníticos são mais sensíveis à compactação que os oxídico-gibbsíticos.

2. $\mathrm{O}$ incremento da microporosidade com a compactação aumentou a retenção de água no solo, mas este aumento não se expressou em termos de conteúdo de água disponível no solo (ADS), exceção para $\theta_{\text {gray }}$ no LA, em que a redução da ADS foi de 48\%, após uma compactação de $900 \mathrm{kPa}$.

3. A condutividade hidráulica e a resistência a penetração foram as propriedades mais sensíveis aos efeitos da compactação.

\section{LITERATURA CITADA}

Arshad, M.A.; Lowery, B.; Grossman, B. Physical tests for monitoring soil quality. In: Doran, J.W.; Jones, A.J. (ed.). Methods for assessing soil quality. Madison: Soil Science Society of America, 1996. p.123-141 (SSSA Special publication 49)

Beutler, A.N.; Silva, M.L.N.; Curi, N.; Ferreira, M.M.; Pereira Filho, I.A.; Cruz, J.C. Agregação de Latossolo Vermelho distrófico típico relacionada com o manejo na região dos cerrados no Estado de Minas Gerais. Revista Brasileira de Ciência do Solo, Viçosa, v.25, n.1, p.129-136, 2001.

Borges, E.N.; Lombardi Neto, F.; Corrêa, G.F.; Costa, L.M. Misturas de gesso e matéria orgânica alterando atributos físicos de um latossolo com compactação simulada. Revista Brasileira de Ciência do Solo, Viçosa, v.21, n.1, p.125-130, 1997.

Bremner, J.M.; Mulvaney, C.S. Nitrogen - Total. In: Page, A.L.; Miller, R.H.; Keeney, D.R. (ed.). Methods of soil analysis, 2.ed. Part 2. Madison: American Society of Agronomy/Soil Science Society of America, 1982. p.595-624.

Cerdà, A. Seasonal variability of infiltration rates under contrasting slope conditions in southeast Spain. Geoderma, Amsterdam, v.69, n.2, p.217-232, 1996.

Curi, N.; Larach, J.O.I.; Kämpf, N.; Moniz, A.C.; Fontes, L.E.F. Vocabulário de ciência do solo. Campinas: Sociedade Brasileira de Ciência do Solo, 1993. 90p.

Dias Júnior, M.S.; Pierce, F.J. O processo de compactação do solo e a sua modelagem. Revista Brasileira de Ciência do Solo, Viçosa, v.20, n.2, p.175-182, 1996.

EMBRAPA - Empresa Brasileira de Pesquisa Agropecuária. Centro Nacional de Pesquisa de Solos. Manual de métodos de análise de solo. 2.ed. Rio de Janeiro: Embrapa Produção de informação, 1997. 212p.

Grant, C.A.; Lafond, G.P. The effects of tillage systems and crop sequences on soil bulk density and penetration resistence on a clay soil in Southern Saskatchewan. Canadian Journal of Soil Science, Ottawa, v.73, n.2, p.223-232, 1993.

Greacen, E.L.; Sands, R. Compaction of forests soil, a review. Australian Journal of Soil Research, Sydney, v.17, n.1, p.163-189, 1980. 
Ishaq, M.; Ibrahim, M.; Hassan, A.; Saeed, M.; Lal, R. Subsoil compaction effects on crops in Punjab, Pakistan: II. Root growth and nutrient uptake of wheat and sorghum. Soil and Tillage Research, Amsterdam, v.60, n.1, p.153-161, 2001.

Kayombo, B.; Lal, R. Responses of tropical crops to soil compaction. In: Soane, B.D.; van Ouwerkerk, C. (ed.). Soil compaction in crop production. Amsterdam: Elsevier, 1994. p.287-316.

Kemper, W.D.; Rosenau, R.C. Aggregate stability and size distribution. In: Klute, A. (ed.). Methods of soil analysis, 2. ed. Part 1 - Physical and mineralogical methods. Madison: American Society of Agronomy/Soil Science Society of America, 1986. p.425-442.

Mapfumo, E.; Chanasyk, D.S.; Naeth, M.A.; Baron, V.S. Forage growth and yield components as influenced by subsurface compaction. Agronomy Journal, Madison, v.90, n.4, p.805-812, 1998.

Marschner, H. Mineral nutrition of higher plants. 2.ed. San Diego: Academic Press, 1995. 889p.

Marsili, A.; Servadio, P.; Pagliai, M.; Vignozzi, N. Changes of some physical properties of a clay soil following passage of rubber- and metal-tracked tractors. Soil and Tillage Research, Amsterdam, v.49, n.2, p.185-199, 1998.

Martino, D.L.; Shaykewich, C.F. Root penetration profiles of wheat and barley as affected by soil penetration resistance in field conditions. Canadian Journal of Soil Science, Ottawa, v.74, n.2, p.193-200, 1994.

McQueen, D.J.; Shepherd, T.G. Physical changes and compaction sensitivity of a fine-textured, poorly drained soil (Typic Endo-aquept) under varying durations of cropping, Manawatu Region, New Zealand. Soil and Tillage Research, Amsterdam, v.63, n.1, p.93-107, 2002.
Nadian, H.; Smith, S.E.; Alston, A.M.; Murray, R.S. The effect of soil compaction on growth and P uptake by Trifolium subterraneum: interactions with mycorrhizal colonisation. Plant and Soil, Dordrecht, v.182, n.1, p.39-49, 1996.

Oliveira, C.V.; Bahia, V.G.; Paula, M.B. Compactação do solo devido à mecanização florestal: causas, efeitos e práticas de controle. Informe Agropecuário, Belo Horizonte, v.191, n.1, p.46-48, 1998.

Oussible, M.; Crookston, P.K.; Larson, W.E. Subsurface compaction reduces the root and shoot growth and grain yield of wheat. Agronomy Journal, Madison, v.84, n.1, p.34-38, 1992.

Pagliai, M.; Febo, P.; La Marca, M.; Lucamonte, G. Effetti del compattamento provocato da differenti tipi di pneumtici su porosità e strutura del terreno. Rivista di Ingegnería Agraria, Bologna, v.3, n.1, p.168-176, 1992.

Silva, A.P.; Libardi, P.L.; Camargo, O.A. Influência da compactação nas propriedades físicas de dois Latossolos. Revista Brasileira de Ciência do Solo, Viçosa, v.10, n.1, p.91-95, 1986.

Silva, M.L.N.; Curi, N.; Blancaneaux, P. Sistemas de manejo e qualidade estrutural de Latossolo Roxo. Pesquisa Agropecuária Brasileira, Brasília, v.35, n.12, p.2485-2492, 2000.

Unger, P.W.; Kaspar, T.C. Soil compaction and root growth: a review. Agronomy Journal, Madison, v.86, n.4, p.759-766, 1994.

Wagger, M.G.; Denton, H.P. Influence of cover crop and wheel traffic on soil physical properties in continuous no till corn. Soil Science Society America Journal, Madison, v.53, n.5, p.1206-1210, 1989.

Yeomans, J.C.; Bremner, J.M. A rapid and precise method for routine determination of organic carbon in soil. Communications in Soil Science and Plant Analysis, Monticello, v.19, n.6, p.1467-1476, 1988. 